\title{
RELAÇÃO ENTRE HABILIDADES DE PROCESSAMENTO AUDITIVO E FUNÇÕES NEUROPSICOLÓGICAS EM ADOLESCENTES
}

\author{
Relation between auditory processing abilities \\ and neuropsychological process in teenagers
}

\author{
Mirella Liberatore Prando ${ }^{(1)}$, Josiane Pawlowski ${ }^{(2)}$, Jandyra Maria Guimarães Fachel ${ }^{(3)}$, \\ Mari Ivone Lanfredi Misorelli ${ }^{(4)}$, Rochele Paz Fonseca ${ }^{(5)}$
}

\begin{abstract}
RESUMO
Objetivo: averiguar a existência de relações entre o desempenho em testes de processamento auditivo e em tarefas cognitivas. Métodos: participaram 12 adolescentes com desenvolvimento típico, estudantes de terceiro ano do Ensino Médio de escolas privadas do estado do Rio Grande do Sul, Brasil, examinados com uma bateria de testes de PA(C) e com o Instrumento de Avaliação Neuropsicológica Breve Neupsilin. Foram realizadas análises de correlação de Pearson e análises qualitativas intertestes para comparação do desempenho na avaliação do $\mathrm{PA}(\mathrm{C})$ e na avaliação neuropsicológica. Resultados: da bateria de testes $\mathrm{PA}(\mathrm{C})$, os Testes SSI - MCI - Teste de Sentenças Sintéticas com mensagem competitiva ipsilateral, SSW - Teste de Dissílabos Alternados e o teste Dicóticos de Dígitos apresentaram correlação forte significativa positiva (coeficiente de correlação de Pearson) com os subtestes do Neupsilin: atenção - contagem inversa, percepção de faces, linguagem oral automática e repetição, memória verbal episódica e memória de trabalho - span auditivo de palavras em sentenças. Alguns testes do PA(C) apresentaram maior frequência de dissociações com subtestes neuropsicológicos. As correlações encontradas indicam que os testes de PA(C) e as tarefas neuropsicológicas parecem examinar algumas habilidades cognitivas subjacentes em comum. As dissociações observadas sugerem que os processamentos auditivo e neuropsicológico são relativamente independentes e a análise intertestes do desempenho dos participantes na avaliação do PA(C), comparada ao desempenho na avaliação neuropsicológica, confirma os resultados encontrados para as questões anteriores. Conclusão: ressalta-se a necessidade de se pensar o contexto da administração dos testes de $\mathrm{PA}(\mathrm{C})$, considerando-se a relação de complementaridade da avaliação neuropsicológica e do exame de processamento auditivo.
\end{abstract}

DESCRITORES: Percepção Auditiva; Testes Neuropsicológicos; Neuropsicologia; Adolescente

(1) Fonoaudióloga; Membro do Grupo de Pesquisa Neuropsicologia Clínica e Experimental, GNCE, Porto Alegre, RS, Brasil; Especialização em Neuropsicologia pela Universidade Federal do Rio Grande do Sul; Mestranda em Psicologia, área de concentração em Cognição Humana da Pontifícia Universidade Católica do Rio Grande do Sul.

(2) Psicóloga; Membro do Grupo de Neuropsicologia Clínica e Experimental, GNCE, Porto Alegre, RS, Brasil; Especialista em Avaliação Psicológica Universidade Federal do Rio Grande do Sul; Mestre em Psicologia pela Universidade Federal do Rio Grande do Sul; Doutoranda em Psicologia na Universidade Federal do Rio Grande do Sul.

(3) Graduada em Matemática; Professora Titular do Departamento de Estatística, Instituto de Matemática e do Programa de Pós-Graduação em Epidemiologia da Universidade Federal do Rio Grande do Sul, UFRGS, Porto Alegre, $\mathrm{RS}$, Brasil; PhD em Estatística pela University of London.
(4) Fonoaudióloga; Docente do CEFAC - Pós-Graduação em Saúde e Educação, São Paulo, SP, Brasil; Mestre em Fonoaudiologia pela Pontifícia Universidade Católica de São Paulo.

(5) Fonoaudióloga e Psicóloga; Professora Adjunta da Faculdade de Psicologia e do Programa de Pós-Graduação em Psicologia, área de concentração Cognição Humana da Pontifícia Universidade Católica do Rio Grande do Sul, PUC-RS, Porto Alegre, RS, Brasil; Coordenadora do Grupo de Pesquisa Neuropsicologia Clínica e Experimental, GNCE, Porto Alegre, RS, Brasil; Doutora em Psicologia do Desenvolvimento pela Universidade Federal do Rio Grande do Sul e Université de Montreal, com Pós-Doutoramento em Neurociências pela Pontifícia Universidade Católica do Rio de Janeiro.

Conflito de interesses: inexistente 


\section{INTRODUÇÃO}

O presente artigo aborda a relação entre o desempenho de adolescentes em habilidades de processamento auditivo (central) e em funções neuropsicológicas. O Processamento Auditivo (Central) $[\mathrm{PA}(\mathrm{C})]$ pode ser definido como o processamento perceptual da informação auditiva pelo sistema nervoso central (SNC) e a atividade neurobiológica subjacente. Inclui mecanismos auditivos relacionados às seguintes habilidades: 1) localização e lateralização do som; 2) discriminação auditiva; 3) reconhecimento de padrão auditivo; 4) aspectos temporais da audição, entre eles, integração temporal, discriminação temporal (por exemplo, detecção de intervalo de tempo), ordenação temporal e mascaramento temporal; 5) desempenho auditivo diante de sinais acústicos competitivos (incluindo escuta dicótica); e 6) desempenho auditivo com degradação de sinais acústicos ${ }^{1}$.

O PA(C) consta de quatro mecanismos auditivos: processamento temporal escuta dicótica, monoaural de baixa redundância e interação binaural. $O$ processamento temporal refere-se ao modo como o sistema nervoso auditivo central (SNAC) analisa aspectos temporais do sinal acústico; é o mecanismo auditivo mais elaborado, pois inclui muitas habilidades auditivas e muitos níveis do SNAC. A habilidade de percepção do contorno do sinal acústico contribui para o reconhecimento dos aspectos de prosódia, como ritmo, tonicidade e entonação. A escuta dicótica caracteriza-se pela condição em que diferentes estímulos são apresentados para cada orelha simultaneamente, situação de escuta em que as vias contralaterais dominam e suprimem as vias ipsilaterais. Avalia a função hemisférica, a transferência inter-hemisférica de informação, maturação e desenvolvimento do SNAC. O mecanismo auditivo monoaural de baixa redundância ocorre quando há a redução da redundância extrínseca do sinal de fala e avalia a habilidade de fechamento auditivo. Essa habilidade é necessária para que o indivíduo complete a informação quando parte dela for perdida durante a redução do sinal de fala. Estão envolvidas nessa função, a discriminação auditiva e a decodificação. A interação binaural refere-se à habilidade do SNAC em receber informações díspares, embora complementares, e unificá-las em um evento perceptual. Este processo depende primariamente da integridade de estruturas do tronco encefálico, além do sistema auditivo periférico. No entanto, podem ser afetados por disfunções/lesões corticais. As funções auditivas que dependem da interação são: localização e lateralização, diminuição de mascaramento binaural, detecção de sinais no ruído e fusão binaural ${ }^{2,3}$. A função auditiva cen- tral envolve muito mais do que um mapa do sistema nervoso central para a porção auditiva. Trata-se de uma dinâmica complexa que envolve transformações do estímulo acústico na cóclea, as vias eferentes, vias de processamento no cérebro além do papel das estruturas fora do lobo temporal posterior como prosencéfalo, por exemplo, para que ocorra os mecanismos ouvir e escutar" ${ }^{4}$.

Dessa forma, algumas habilidades envolvidas no processamento auditivo (central) [PA(C)] são específicas da modalidade auditiva e outras dependem de processos cognitivos adicionais ${ }^{2,5}$. Inúmeras discussões a cerca do entendimento do processamento auditivo como modalidade específica e o diagnóstico diferencial vêm ocorrendo na tentativa de elucidar o conceito dos seus distúrbios ${ }^{5-7}$. Algumas definições de $[\mathrm{PA}(\mathrm{C})]$ consideram que o diagnóstico do transtorno do processamento auditivo (central) [TPA(C)] pode ser aplicado somente quando um déficit (perceptual) é demonstrado no sistema auditivo ${ }^{6-8}$. Outros autores, apoiados na revisão da literatura em neurociência, neurofisiologia cognitiva e auditiva e áreas afins, afirmam que a definição de TPA(C), a qual inclui modalidade específica como critério diagnóstico, é neurofisiologicamente insustentável ${ }^{1,5,9}$.

Entende-se, portanto, que exista uma relação entre processamento auditivo (central) e as habilidades neuropsicológicas, contudo, a identificação de quais processos cognitivos estariam envolvidos no processamento auditivo ainda não é consensual na literatura e pode ser considerada, até mesmo, pouco explorada. Alguns autores argumentam que, devido à natureza complexa dos testes propostos para a avaliação de $\mathrm{PA}(\mathrm{C})$, os resultados podem sofrer a interferência dos aspectos neuropsicológicos e que o dado encontrado de $\mathrm{PA}(\mathrm{C})$ poderia ser um sintoma dentro de um quadro maior ${ }^{7,8}$. Outros afirmam que mesmo considerando o fato de que a investigação dos aspectos perceptuais auditivos possa sofrer influência de outros fatores, é possível diferenciar um déficit mais global (ou supramodal) de uma dificuldade mais específica de $\mathrm{PA}(\mathrm{C}){ }^{9}$.

Considerando essa abordagem, o PA(C) também poderia ser considerado uma modalidade envolvida nas funções neuropsicológicas ${ }^{10}$. A avaliação neuropsicológica deve envolver a verificação das funções sensoriais receptivas e perceptivas, que devem ser analisadas conjuntamente com os demais aspectos cognitivos, tais como memória, aprendizado, pensamento, atenção, funções expressivas, linguagem e funções executivas. No entanto, a percepção auditiva é menos investigada numa avaliação neuropsicológica do que a percepção visual ${ }^{11}$. 
A avaliação neuropsicológica é um método de examinar as funções cognitivas através do estudo da expressão comportamental das disfunções cerebrais ${ }^{11}$. A avaliação comportamental do PA(C) pode ser realizada a partir do exame das habilidades auditivas centrais que são eliciadas através de testes específicos. Através da aplicação desses testes, em conjunto com a avaliação neuropsicológica, é possível verificar a existência de correlações e dissociações no desempenho do paciente nas avaliações. O conceito de dissociação é utilizado na clínica neuropsicológica em busca do entendimento dos déficits cognitivos. Segundo Willmes ${ }^{12}$, a dissociação pode ser definida como uma diferença no desempenho de um paciente em duas diferentes tarefas ou, ainda, os prejuízos de desempenho de algumas tarefas em contraposição a um desempenho normal em outras tarefas, são apontados como evidência da existência de diferentes sistemas neurais subjacentes ${ }^{10}$. Devido à forte natureza desse modelo de pesquisa, experimentos de dissociação estão tornando-se mais prevalentes ${ }^{7}$. Em virtude da lacuna na literatura específica sobre as relações entre processamento auditivo e habilidades cognitivas, torna-se relevante investigar empiricamente se tais relações existem e, caso existam, como se dão.

A partir dessa visão integradora entre a avaliação de processamento auditivo e de funções cognitivas, o objetivo geral desse estudo foi investigar a existência de relações entre o desempenho em $\mathrm{PA}(\mathrm{C})$ e em tarefas neuropsicológicas. Pretendeuse, então, responder às seguintes questões de pesquisa:

1) Há correlação entre o desempenho em $P A(C)$ e o desempenho neuropsicológico em uma amostra de adolescentes saudáveis?

2) Podem ser observadas dissociações de desempenho nas tarefas de PA(C) e nas tarefas de orientação têmporo-espacial, atenção, percepção visual, memória, habilidades aritméticas, linguagem, praxias e funções executivas em adolescentes?

3) A análise intertestes do desempenho dos participantes na bateria selecionada para a avaliação do $\mathrm{PA}(\mathrm{C})$, quando comparada ao desempenho na avaliação neuropsicológica, confirma os resultados encontrados para as questões anteriores?

Para tais questões norteadoras, formularam-se as seguintes hipóteses:

1) Haverá correlações entre tarefas de $P A(C)$ e tarefas de avaliação neuropsicológica, principalmente com subtestes de atenção e de memória.

2) Serão observadas dissociações entre o desempenho nos testes de $\mathrm{PA}(\mathrm{C})$ e o desempenho em alguns subtestes do exame neuropsicológico, tais como, desempenho de processamento preservado acompanhado de desempenho deficitário em subtestes neuropsicológicos, por exemplo, orientação têmporo-espacial e praxias, ou vice-versa.

3) A análise intertestes da avaliação do $\mathrm{PA}(\mathrm{C})$, comparada à análise do desempenho neuropsicológico, confirmará as duas hipóteses anteriores.

\section{MÉTODOS}

Participaram do estudo 12 adolescentes, sete do sexo feminino e cinco do masculino, de 16 (16,67\%) e $17(83,3 \%)$ anos de idade, todos com escolaridade de 10 anos completos de estudo formal e o terceiro ano do Ensino Médio em curso, em escolas privadas do Estado do Rio Grande do Sul. A frequência de hábitos de leitura e escrita variou de no mínimo uma vez por semana a todos os dias. Em sua maioria, os participantes pertenciam às classes econômicas B1 (50\%) e A2 (41,7\%), sendo apenas um da classe $A 1$, segundo o Critério de Classificação Econômica Brasil - CCEB ${ }^{13}$.

No que diz respeito aos critérios de inclusão na amostra participaram apenas brasileiros natos, com fluência no Português Brasileiro, sem história prévia ou atual de quaisquer distúrbios neurológicos, psiquiátricos, visuais, auditivos e/ou linguísticos auto-relatados, de alcoolismo ou de uso de drogas ilícitas ou de benzodiazepínicos.

De acordo com os princípios éticos das pesquisas com seres humanos, os participantes foram avaliados voluntariamente, mediante autorização através da assinatura do Termo de Consentimento Livre e Esclarecido, pela diretoria da instituição educacional, por um de seus responsáveis legais e por ele/ela próprio(a).

Os participantes foram examinados por um fonoaudiólogo em duas sessões individuais de uma hora, em média, nas quais foram submetidos à avaliação auditiva periférica e central. A avaliação auditiva periférica envolveu audiometria tonal, índice percentual de reconhecimento de fala e limiar de reconhecimento de fala e imitanciometria, composta de timpanograma e pesquisa de reflexos ipsi e contralaterais. Todos os participantes incluídos obtiveram limiares auditivos dentro da normalidade na audiometria tonal, presença de reflexos acústicos e funcionamento normal de orelha média.

A avaliação auditiva central foi composta por testes gravados em compact disc (CD) e comercializados pela Auditec - St. Louis ${ }^{14}$ e por Pereira e Schochat ${ }^{15}$. Os mecanismos avaliados foram escuta dicótica, interação binaural, processamento temporal e monoaurais de baixa redundância. A 
análise da bateria permitiu a caracterização da função auditiva central, compreendida entre tronco encefálico e o córtex auditivo, possibilitando a caracterização da função auditiva (envolvendo as habilidades auditivas) como normal ou alterada. Os testes incluídos na bateria foram os que seguem, descritos brevemente:

A) Testes dicóticos: Teste Dicótico de Dissílabos Alternados (SSW) e Dicótico de Dígitos (DD). Ambos avaliam o mecanismo de escuta dicótica por meio da apresentação simultânea de estímulos diferentes em cada orelha.

O SSW utiliza como estímulo verbal palavras dissílabas. São 40 itens compostos por quatro dissílabas cada um, o que totaliza 160 palavrasestímulo. Os 20 itens de números ímpares são apresentados, iniciando-se pela orelha direita, e os 20 pares, iniciando-se pela orelha esquerda. A primeira palavra dos itens ímpares é apresentada sozinha à orelha direita e constitui a condição direita não-competitiva. Em seguida, são apresentadas duas palavras diferentes, uma para cada uma das orelhas, simultaneamente, formando a condição direita e esquerda competitiva. A última palavra é apresentada isolada à orelha esquerda, formando a condição esquerda não-competitiva. Os itens pares seguem os mesmos critérios, só que se iniciam pela orelha esquerda.

O DD envolve a apresentação de uma lista de 20 pares de dígitos dissílabos. É solicitada tanto a tarefa de integração binaural, quando o indivíduo repete os estímulos ouvidos em ambas as orelhas, e a de separação binaural, quando o indivíduo repete o estímulo de apenas uma orelha. Estes testes foram apresentados em um nível de 50 decibéis nível de sensação (dBNS), tendo como base os limiares médios tonais.

B) Teste de processamento temporal: Teste de Reconhecimento do Padrão de Frequência (PPST). Avalia o mecanismo de processamento temporal mediante a apresentação de uma sequência de três tons não-verbais que variam em frequência. É solicitado ao indivíduo que inicialmente murmure as sequências ouvidas e depois as nomeie. Esse teste foi apresentado em um nível de $50 \mathrm{dBNS}$, tendo como base os limiares médios tonais.

C) Teste monoaural de baixa redundância: Teste de Identificação de Sentenças Sintéticas (SSI). Avalia as habilidades de fechamento auditivo, figura-fundo e discriminação, quando uma parte do sinal auditivo está distorcida ou ausente e/ou em presença de estímulos auditivos competitivos (história). É solicitado ao indivíduo que despreze a mensagem competitiva e indique as frases ouvidas que estão escritas em uma folha. Foi apresentado na modalidade de mensagem competitiva ipsilateral (MCI). $O$ teste foi aplicado em um nível de $40 \mathrm{dBNS}$ nas relações estímulo/competição de $0 \mathrm{~dB},-10 \mathrm{~dB}$ e $-15 d B$.

D) Teste de interação binaural: Teste de diferença de Limiar de Mascaramento (MLD). Examina a habilidade do sistema nervoso auditivo central para processar informação díspar, mas complementar, apresentada às duas orelhas. É solicitado ao indivíduo que despreze a presença do ruído mascarador e indique a presença de um tom pulsátil, levantando a mão sempre que o perceber. Este teste foi aplicado em um nível de 40 dBNS.

Além da avaliação auditiva periférica e central, os participantes responderam aos seguintes instrumentos:

E) Questionário estruturado de dados socioculturais e aspectos da saúde ${ }^{16}$. Inclui a avaliação de questões referentes à renda, hábitos culturais e comunicativos e antecedentes médicos (aspectos de saúde geral, sensorial e neurológica). Este instrumento inclui a Escala CAGE ${ }^{17}$ para avaliação dos hábitos de ingestão de bebida alcoólica. Tais avaliações confirmaram os critérios de inclusão no estudo.

F) Instrumento de Avaliação Neuropsicológica Breve Neupsilin ${ }^{18,19}$. Fornece um perfil neuropsicológico breve, delimitando, em aproximadamente 45 minutos, a presença ou ausência de déficit nas funções cognitivas: orientação têmporo-espacial, atenção (concentrada), percepção (visual), memória (cinco sistemas), habilidades aritméticas, linguagem (oral e escrita), praxias e funções executivas (resolução de problemas e fluência verbal), avaliadas por meio de 32 subtestes. Permite, também, qualificar tipos de erros e algumas estratégias de processamento utilizadas pelo avaliando. Os subtestes são apresentados abaixo, conforme a função neuropsicológica investigada.

1) Orientação Têmporo-espacial: Tempo: resposta de dia da semana, dia do mês, mês e ano, e Espaço: resposta de local, cidade, estado e país;

2) Atenção: 1) Contagem Inversa: contagem de 50 a 30 pelo examinando e contagem do tempo de realização da tarefa pelo examinador para avaliar a velocidade de processamento e 2) Repetição de Sequência de Dígitos: repetição de uma sequência de sete números;

3) Percepção: 1) Verificação de Igualdade e Diferença de Linhas: verificação de seis pares de linhas; 2) Heminegligência Visual: percepção de todo espaço de uma folha de papel repleta de traços que deverão ser riscados; 3) Percepção de Faces: avaliação, como iguais ou diferentes, de três pares de fotografias de faces, sendo uma delas de frente e outra de perfil; e 4) Reconhecimento de Faces: memorização de dois rostos desenhados, que devem 
ser reconhecidos entre quatro apresentados posteriormente;

4) Memória: 1) Memória de Trabalho: a) Ordenamento Ascendente de Dígitos: repetição em ordem crescente de 10 conjuntos de dois até seis dígitos; b) Span Auditivo de Palavras em Sentenças: memorização e evocação das últimas palavras de frases, após a leitura de conjuntos de dois, três, quatro e cinco sentenças pelo examinador; 2) Memória Verbal Episódico-Semântica: a) Evocação Imediata: repetição de nove palavras ditas pelo examinador, $b$ ) Evocação Tardia: emissão das mesmas nove palavras em tempo posterior, e c) Reconhecimento: indicação, em uma lista de 18 palavras, da presença ou não da palavra na lista original falada pelo examinador; 3) Memória Semântica de Longo Prazo: resposta a duas perguntas referentes a conhecimentos gerais; 4) Memória Visual de Curto Prazo (três estímulos): memorização de uma figura sem sentido de cada vez e reconhecimento entre um conjunto de três figuras semelhantes; 5) Memória Prospectiva: lembrança, ao final da testagem, da instrução de escrever o nome em uma folha de papel fornecida no início da avaliação;

5) Habilidades Aritméticas: resolução de quatro cálculos, um de cada operação aritmética básica;

6) Linguagem: 1) Linguagem Oral: a) Nomeação de dois objetos e de duas figuras; b) Repetição de oito palavras reais e de duas pseudopalavras; c) Linguagem Automática: contagem de um a dez e verbalização de todos os meses do ano, em ordem; d) Compreensão Oral (três conjuntos de figuras): indicação da figura correspondente ao enunciado verbal do examinador; e) Processamento de Inferências: explicação do significado de um provérbio e de duas metáforas; 2) Linguagem Escrita: a) Leitura em Voz Alta de dez palavras reais e de duas pseudopalavras; b) Compreensão Escrita (três estímulos): leitura em silêncio de palavras e frases e indicação das figuras correspondentes; c) Escrita Espontânea de uma frase; d) Escrita Copiada de uma frase, e e) Escrita Ditada de dez palavras reais e de duas pseudopalavras;

7) Praxias: a) Ideomotora: realização de três gestos, conforme instrução verbal do examinador; b) Construtiva: cópia de três figuras (quadrado, flor e cubo) e desenho de um relógio sem modelo; c) Reflexiva: repetição de uma sequência de três gestos;

8) Funções executivas: 1) Resolução de problemas: resposta a duas perguntas envolvendo raciocínio abstrato; 2) Fluência Verbal fonêmica: ver- balização, durante um minuto, de palavras que iniciem com a letra $\mathrm{F}$.

A pesquisa foi aprovada pelo Comitê de Ética em Pesquisa da Universidade Federal do Rio Grande do Sul (parecer 2008/09).

Os resultados obtidos nos testes de $\mathrm{PA}(\mathrm{C})$ foram analisados descritivamente, em forma de percentual de acerto. Do mesmo modo, os escores brutos obtidos no Neupsilin foram convertidos para percentual. Além disso, efetuou-se uma análise de ocorrência de déficits a partir dos dados normativos nacionais, para a verificação de dissociações. Por fim, realizou-se uma análise subjetiva global (interpretação intertestes) do perfil geral de processamento e de desempenho neuropsicológico, mediante o julgamento de dois fonoaudiólogos, com domínio da teoria e do método de avaliação de processamento auditivo, e de dois neuropsicólogos, com domínio dos pressupostos da avaliação neuropsicológica. Ambos julgamentos foram independentes, com estabelecimento de consenso quando necessário.

Os dados foram submetidos a análises descritivas (média e desvio-padrão) e à análise de Correlação de Pearson para estudar a associação do desempenho dos adolescentes nas habilidades de processamento auditivo e nas tarefas neuropsicológicas. Foram, ainda, agrupados conforme frequência de déficits.

\section{RESULTADOS}

Os resultados serão apresentados em três sessões: 1) dados descritivos da amostra e análise correlacional; 2) dissociações e 3) análise comparativa inter e intratestes (PA(C) versus Neupsilin).

\section{Dados descritivos da amostra e análise correlacional}

$\mathrm{Na}$ Tabela 1, apresentam-se as médias de percentuais de acertos nos testes de PA(C), com seus respectivos desvios-padrão. Em complementaridade, na Tabela 2, expõem-se as médias de percentuais de acertos nos subtestes do Neupsilin, acompanhados dos desvios-padrão.

Em geral, observando-se os dados na Tabela 1, nota-se que os adolescentes obtiveram um bom desempenho médio na avaliação de $P A(C)$. No MLD, inclusive, não houve erros. Tabela 2: Médias de percentuais de acertos nos subtestes do Neupsilin.

Embora a análise da Tabela 2 evidencie um bom desempenho neuropsicológico da amostra, nota-se que o percentual de acertos foi inferior aos demais em sustestes que examinam atenção, memória e funções executivas. 
Tabela 1 - Percentuais de acertos nos testes de avaliação do PA(C)

\begin{tabular}{|c|c|c|}
\hline Testes PA(C) & \multicolumn{2}{|c|}{$\begin{array}{l}\text { Médias dos percentuais de acertos } \\
\text { (Desvio-padrão) }\end{array}$} \\
\hline MLD & \multicolumn{2}{|c|}{$100,00(0,00) \%$} \\
\hline PPST & & \\
\hline Etapa Imitação & \multicolumn{2}{|c|}{$88,88(22,77) \%$} \\
\hline Etapa Nomeação & \multicolumn{2}{|c|}{$86,90(15,19) \%$} \\
\hline SSI & OD & OE \\
\hline Rel. $s / r=0$ & $89,17(15,05) \%$ & $89,17(10,83) \%$ \\
\hline SSW & $\mathrm{DC}$ & EC \\
\hline \% acertos condições competitivas & $95,21(5,05) \%$ & $92,50(7,76) \%$ \\
\hline \multicolumn{3}{|l|}{ Dicótico de Dígitos } \\
\hline Etapa Integração Binaural & OD & OE \\
\hline \multirow{3}{*}{ Etapa Separação Binaural } & $99,58(1,44) \%$ & $99,58(1,44) \%$ \\
\hline & $A D O D$ & AD OE \\
\hline & $99,17(2,88) \%$ & $98,88(2,70) \%$ \\
\hline
\end{tabular}

Rel. $s / r=$ sinal/ruído; $O D=$ orelha direita; $O E=$ orelha esquerda; $D C=$ direita competitiva; $E C=$ esquerda competitiva; $A D$ OD = atenção dirigida a OD; $A D O E$ = atenção dirigida a OE.

Tabela 2 - Percentuais de acertos nas funções neuropsicológicas avaliadas pelo Neupsilin

\begin{tabular}{lc}
\hline Funções e componentes avaliados & Média de percentuais de acertos \\
\hline 1. Orientação têmporo-espacial & $100(0,00)$ \\
1.1 Tempo & $100(0,00)$ \\
1.2. Espaço & $100(0,00)$ \\
2) Atenção (concentrada) & $91,58(8,46)$ \\
2.1) Contagem inversa & $99,58(1,44)$ \\
2.2) Repetição de seqüência de dígitos & $67,50(30,53)$ \\
3) Percepção & $90,97(13,51)$ \\
3.1) Verificação de igualdade e diferença de linhas & $90,2758(13,21)$ \\
3.2) Heminegligência visual & $100(0,00)$ \\
3.3) Percepção de faces & $86,11(26,43)$ \\
\hline
\end{tabular}

Na Figura 1, pode-se visualizar os resultados da análise correlacional entre os escores dos subtestes do Neupsilin e do exame de PA(C). Os gráficos expostos na Figura 1 mostram apenas as correlações significantes.

Observa-se que, dentre as correlações significantes encontradas, todas foram positivas e fortes, exceto a correlação entre o teste dicótico de dígitos e o subteste do Neupsilin evocação imediata da memória episódico-semântica.

\section{Dissociações}

$\mathrm{Na}$ Tabela 3, expõe-se a quantidade de dissociações, totalizando dois tipos na comparação de cada prova de $P A(C)$ e do Neupsilin: 1) presença de déficit em um dos testes de $\mathrm{PA}(\mathrm{C})$ e ausência de déficit em um dos subtestes do Neupsilin e 2) ausência de déficit em um dos testes de $\mathrm{PA}(\mathrm{C})$ e presença de déficit em um dos subtestes do Neupsilin.

Quanto às dissociações encontradas, observouse que alguns testes do $\mathrm{PA}(\mathrm{C})$ apresentam maior frequência de dissociações com subtestes neuropsicológicos. Conforme apresentado na Tabela 3 , o maior número de dissociações com os testes neuropsicológicos foi do Teste de PA(C) SSI/ $\mathrm{MCl}$, seguido, em ordem decrescente, pelos testes, PPST, SSW, DD e MLD. A função neuropsicológica que apresentou mais dissociação com os testes $\mathrm{PA}(\mathrm{C})$ foi: habilidades aritméticas, seguida, em ordem decrescente, pelos subtestes processamento de inferências, percepção (visual), atenção, resolução de problemas, memória de trabalho, 


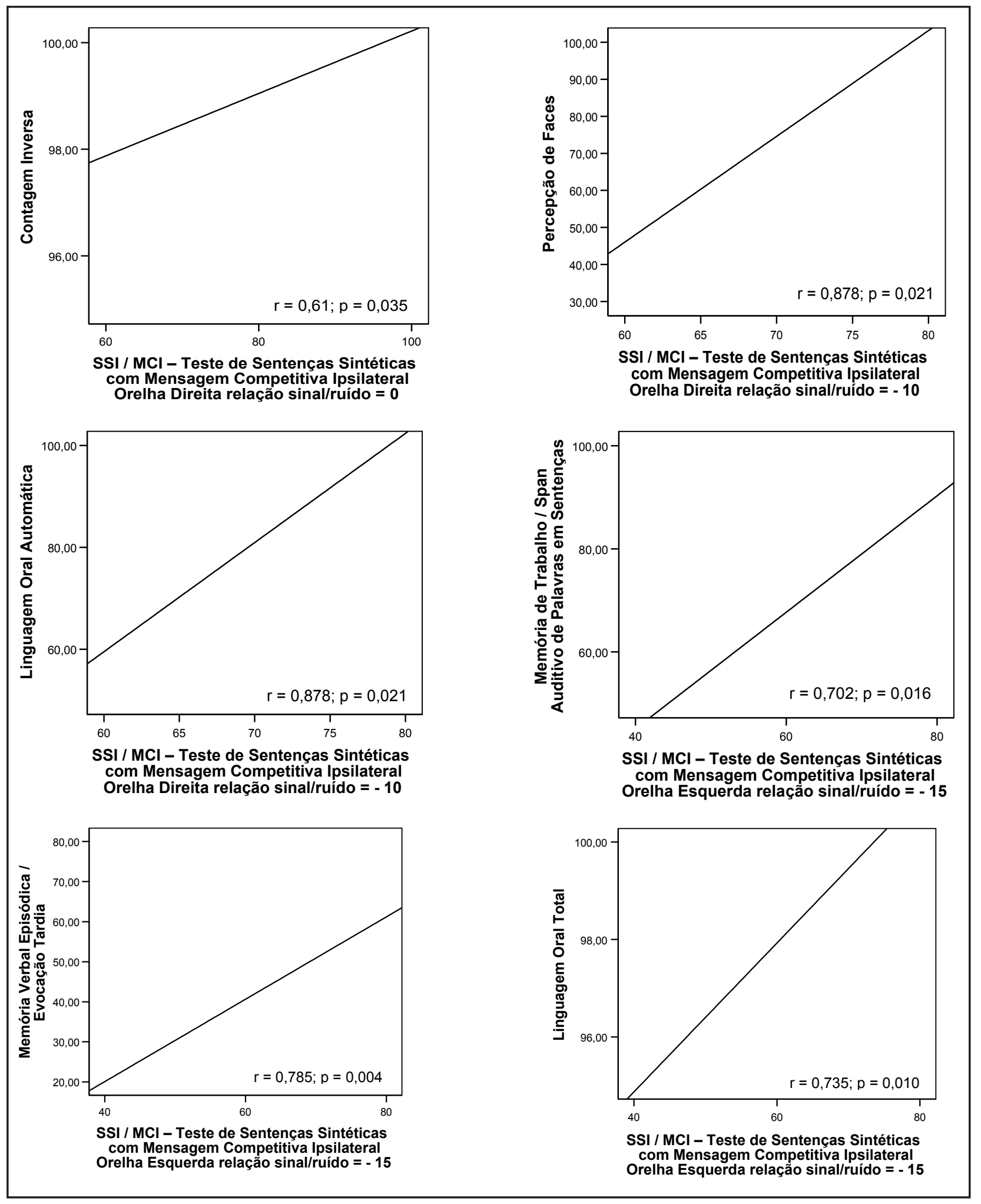




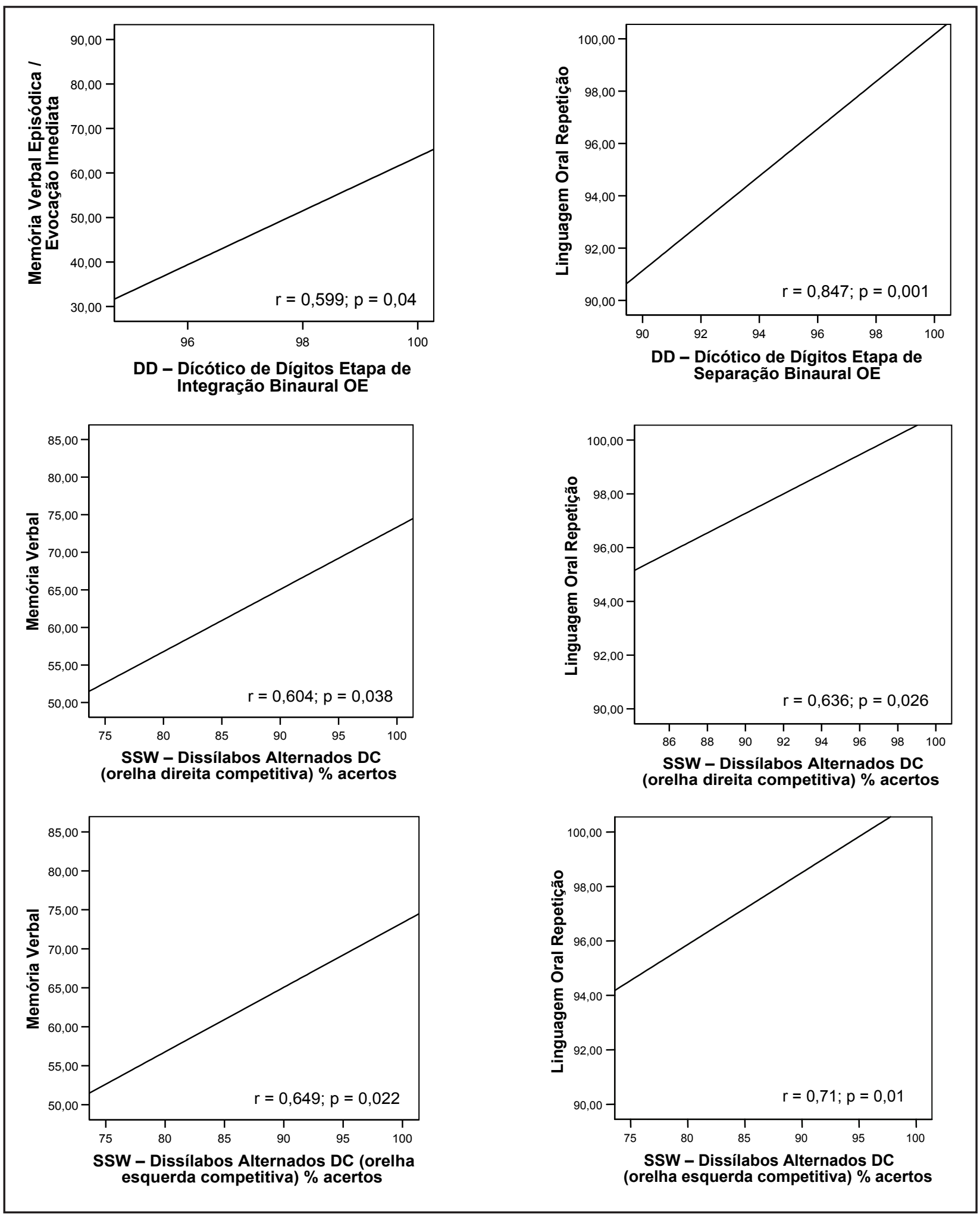

Figura 1 - Gráficos de correlações entre os testes de PA(C) e os subtestes do Neupsilin 
Tabela 3 - Dissociações encontradas entre os testes de PA(C) e os subtestes Neupsilin

\begin{tabular}{lcccccc}
\hline Testes PA(C) & \multicolumn{7}{c}{ Quantidade de dissociações } \\
\cline { 2 - 7 } Subtestes Neupsilin & MLD & PPST & SSI/MCI & SSW & DD & Total \\
\hline Atenção & 02 & 03 & 06 & 05 & 02 & 19 \\
Percepção & 02 & 05 & 06 & 05 & 02 & 20 \\
Memória de trabalho & 02 & 03 & 07 & 04 & 02 & 18 \\
Memória verbal episódica & 01 & 04 & 10 & 01 & 01 & 17 \\
Habilidades aritméticas & 02 & 05 & 10 & 04 & 02 & 23 \\
Linguagem oral repetição & 01 & 04 & 06 & 0 & 01 & 12 \\
Linguagem oral automática & 01 & 06 & 08 & 01 & 01 & 17 \\
Linguagem PI & 03 & 08 & 06 & 02 & 03 & 22 \\
Praxia reflexiva & 02 & 03 & 07 & 03 & 02 & 17 \\
FE resolução de problemas & 03 & 03 & 08 & 02 & 03 & 19 \\
FE fluência verbal & 01 & 04 & 08 & 01 & 01 & 15 \\
Total & 21 & 48 & 76 & 28 & 20 & - \\
\hline
\end{tabular}

$\mathrm{PI}=$ processamento de inferências; $\mathrm{FE}=$ funções executivas.

memória verbal episódica, linguagem oral automática, praxia reflexiva, fluência verbal e linguagem oral repetição.

Salienta-se que nos testes comportamentais de $\mathrm{PA}(\mathrm{C}), \mathrm{MLD}$ e DD, nenhum dos 12 participantes apresentou déficit nas habilidades auditivas examinadas. No entanto, houve ocorrência de déficit da amostra em algumas funções neuropsicológicas avaliadas pelo Neupsilin.

\section{Análise comparativa inter e intratestes (PA(C) versus Neupsilin)}

Foi realizada uma análise inter e intratestes de PA(C) para analisar o perfil geral de desempenho dos participantes. Dos 12 participantes avaliados, seis apresentaram um conjunto de respostas que merecem algumas considerações. Quatro destes seis obtiveram déficits em alguns testes de $P A(C)$, mas a análise do conjunto de resultados encontrados não é compatível com o conjunto de critérios mínimos para o diagnóstico de um Transtorno de Processamento Auditivo (Central) $[\mathrm{TPA}(\mathrm{C})]^{1}$. Os resultados encontrados podem sugerir outro tipo de dificuldade. Esta análise do perfil encontrado foi confrontada com o resultado nas tarefas do Neupsilin e uma análise geral de desempenho em todo o instrumento, visando ao entendimento das respostas. Dos quatro adolescentes cujos resultados na bateria de testes de $\mathrm{PA}(\mathrm{C})$ não foram compatíveis com uma disfunção auditiva central, dois apresentaram alguns déficits em subtestes do Neupsilin, que sugerem dificuldades de atenção. Os outros dois não apresentaram este indício, pois não tive- ram déficits que pudessem sugerir algum tipo de dificuldade cognitiva específica.

Além destes quatro participantes com perfil de $\mathrm{PA}(\mathrm{C})$, alvo de algumas considerações, outros dois participantes apresentaram resultados compatíveis com déficit de PA(C). Esses dados foram obtidos a partir da análise intertestes e de auto-relato de dificuldades em manter a atenção, mas em grau leve, pois nunca procuraram investigar esse sintoma. $\mathrm{Na}$ análise do desempenho nos subtestes do Neupsilin, esses dois participantes não apresentaram déficits que confirmassem um quadro sugestivo de dificuldade de atenção ou em outras funções, sugerindo uma confirmação do déficit perceptual auditivo.

\section{DISCUSSÃO}

No âmbito da interface subjetivamente identificada, mas ainda pouco explorada, entre a avaliação de $\mathrm{PA}(\mathrm{C})$ e o exame neuropsicológico, o objetivo geral desse estudo foi averiguar a existência de relações entre o desempenho em testes de processamento auditivo e em tarefas cognitivas. Foram feitas análises em busca de respostas preliminares a três questões de pesquisa, para as quais se elaboraram hipóteses. A presente discussão será norteada por estes três conjuntos de problema de pesquisa e hipótese.

No que diz respeito à primeira questão, "Há correlação entre o desempenho em $\mathrm{PA}(C)$ e o desempenho neuropsicológico em uma amostra de adolescentes saudáveis?", hipotetizou-se que haveria correlações entre algumas tarefas de processamento auditivo e subtestes do Neupsilin, 
predominantemente com tarefas de atenção concentrada e de memória (de trabalho e episódico-semântica verbal). Os resultados do estudo demonstraram correlações fortes e significantes entre o desempenho de adolescentes em testes que avaliam o processamento auditivo e em tarefas que examinam habilidades neuropsicológicas, principalmente as que investigam atenção concentrada, percepção de faces, linguagem oral e memória (de trabalho e episódico-semântica), confirmando o que se esperava. As correlações fortes podem ser justificadas pelo compartilhamento de habilidades cognitivas subjacentes às tarefas de $\mathrm{PA}(\mathrm{C})$ e destas funções neuropsicológicas. Esse achado corrobora os estudos que demonstram uma relação evidente entre a percepção e produção da linguagem ${ }^{20}$.

A neurociência cognitiva demonstrou que há poucas, se é que existem, áreas inteiramente compartimentadas no cérebro que sejam responsáveis somente por uma única modalidade sensorial. Há evidências de que o processamento de dados sensoriais é interdependente e integrado com base em domínios cognitivos, mais precisamente em atenção, memória e representações linguísticas ${ }^{1}$. Apesar de existirem algumas regiões do cérebro que são específicas para estímulos de modalidade auditiva, a organização cerebral é predominante não modular e não exclusivamente segregada ${ }^{9}$. Algumas definições sugerem que o diagnóstico de TPA(C) deveria ser aplicado somente quando um déficit (perceptual) fosse verificado no sistema auditivo e em nenhuma outra parte ${ }^{8}$. No entanto, a exigência do processamento auditivo como uma modalidade específica para o diagnóstico não deveria ser um critério, pois não é consistente com a forma em que esse tipo de processamento ocorre no SNC 1,5,9.

Mais especificamente, algumas correlações entre subtestes do PA(C) e do Neupsilin serão comentadas. Neste sentido, a correlação significativa forte e positiva entre o teste SSI e a tarefa de atenção - contagem inversa pode ser justificada pela demanda de um alto nível de atenção, concentração e controle mental requeridos no teste. A habilidade avaliada no SSI é a figura-fundo auditiva (identificação de mensagem primária na presença de sons competitivos) diante de estímulos auditivos e visuais. Portanto, é necessário, para a execução do teste, um alto nível de atenção focalizada ou seletiva além da atenção sustentada (mantida/concentrada). Muitas das áreas auditivas são também responsáveis pela manutenção da atenção, controle executivo, regulação motora, estando relacionadas à fisiopatologia do Transtorno do Déficit de Atenção e Hiperatividade $(\mathrm{TDA} / \mathrm{H})^{3}$. Vias auditivas presentes no corpo caloso, responsáveis pela transferência inter-hemisférica desempenham um papel no controle atencional e na sua modulação, além da sua atividade sensorial ${ }^{21}$. Fica clara, assim, a relação estreita entre atenção concentrada auditiva e a habilidade de figura-fundo auditiva. Em complementaridade, houve correlação do teste SSI ainda com um subteste de percepção visual e com tarefas mnemônicas. A correlação do SSI com o teste percepção de faces faz sentido pelo envolvimento da questão auditivo-visual, já que a identificação dos símbolos gráficos (a escrita) é requerida, além da atenção seletiva concentrada. No que tange às associações com a memória, a tarefa Span auditivo de palavras em sentenças, subteste que examina a memória de trabalho, apresentou correlação forte e significativa com o teste SSI (OE na relação-15). É possível inferir, através desse achado, que a dupla tarefa exigida nesta prova do Neupsilin (repetição de frases e armazenamento da última palavra de cada frase, simultaneamente, para posterior evocação dos últimos vocábulos, em ordem), pode apresentar relação com o SSI por esse também requerer a atividade do executivo central. Embora o SSI demande predominantemente atenção focalizada concentrada, para que esta seja processada, é necessária inibição. Na medida em que no teste SSI, o indivíduo escuta uma história narrada enquanto deve ficar alerta para o aparecimento súbito de uma sentença, sabe-se que a tarefa demanda alerta e atenção seletiva. Tais correlações eram, então, esperadas. Essa associação que ocorreu na relação -15 é a mais difícil, demandando ainda mais da atenção e do executivo central, cuja complexidade provavelmente explique a demanda do executivo central na tarefa. Segundo o modelo de memória de trabalho de Baddeley ${ }^{22}$, este sistema mnemônico exige a participação de um supervisor atencional, o executivo central para garantir entrada e a manipulação de dois estímulos simultaneamente.

De um modo geral, quanto ao SSI, a maior ocorrência de correlações se deu com a etapa monoaural OD (orelha direita) e o mesmo não ocorreu com a esquerda. Este fato pode ser justificado por ter sido a OD a primeira orelha testada em todos os participantes, não havendo alternância, por isso é possível hipotetizar que a $\mathrm{OE}$, que foi a segunda, possa ter sofrido efeito de aprendizagem ou de habituação com a situação de avaliação.

No que tange ao SSW, a correlação forte significativa positiva entre este teste e os subtestes de Memória verbal episódico-semântica e Linguagem oral repetição pode ser justificada pelo fato de o SSW envolver a codificação e o armazenamento de curto prazo de estímulos verbais, ao ser solicitada a evocação de quatro palavras na ordem em que foram escutadas, tal como uma prova neuropsicológica de span verbal ${ }^{23}$. Tal correlação era 
esperada, na medida em que em ambos os casos há componentes de memória episódica verbal de curto prazo, além da repetição oral. No entanto, também seria esperada alguma correlação com a memória de trabalho, já que parte de duas palavras foram ouvidas simultaneamente durante a apresentação dicótica, solicitando também atenção dividida (executivo central). Essa correlação não foi, entretanto, encontrada. É possível inferir que a integração binaural, habilidade auditiva envolvida no teste, no momento em que há a apresentação dicótica das duas palavras, esteja mais fortemente envolvida na tarefa, do que a memória de trabalho em si. Cabe ressaltar aqui o apontamento na literatura da importância de se examinar tanto a memória verbal episódica quanto as habilidades auditivas requeridas no SSW. Tal exame é relevante para o diagnóstico diferencial entre TPA $(\mathrm{C})$ e/ou déficit cognitivo mais amplo, tal como o mnemônico. Por exemplo, se o desempenho em memória verbal for muito superior ao desempenho no SSW, na condição de escuta dicótica, é possível inferir que a dificuldade está na habilidade auditiva de integração binaural e não em memória verbal episódica. Caso as dificuldades se apresentem em ambos os testes, maiores investigações dos aspectos cognitivos devem ser consideradas antes do diagnóstico de DPA(C) ser estabelecido ${ }^{2}$.

Frente a estes diagnósticos, a princípio paralelos, salienta-se o cuidado na avaliação de $\mathrm{PA}(\mathrm{C})$ quanto aos resultados serem coerentes e a importância de uma análise inter e intratestes cuidadosa. Resultados incoerentes nos testes ou achados reduzidos pela aplicação restrita da bateria dos testes, sem que outras funções sejam investigadas, não podem conduzir a um diagnóstico de TPA(C) ${ }^{1}$. O potencial para o envolvimento de fatores não diretamente perceptuais no resultado do teste não significa que a percepção auditiva nunca seja um determinante principal de desempenho, mas somente que os resultados não podem ser determinados sem uma investigação posterior ou complementar das demais funções neuropsicológicas ${ }^{2}$. Bellis ${ }^{2}$ afirma que a investigação de um possível TPA(C) deveria ser realizada somente depois que medidas sobre os aspectos cognitivos, de aprendizagem, de fala e de linguagem (oral e escrita) tenham sido obtidas. Em concordância, os pesquisadore ${ }^{1}$ afirmam que quando há prejuízos de fala e linguagem, intelectuais ou psicológicos, a avaliação desses aspectos deve preceder a avaliação do processamento auditivo.

Além da análise correlacional acima discutida, uma segunda questão norteou a presente pesquisa: "Podem ser observadas dissociações de desempenho nas tarefas de $\mathrm{PA}(\mathrm{C})$ e nas tarefas de orien- tação têmporo-espacial, atenção, percepção visual, memória, habilidades aritméticas, linguagem, praxias e funções executivas em adolescentes?". Como resposta a essa questão, esperavam-se dissociações entre o desempenho de processamento auditivo e algumas tarefas neuropsicológicas, concordantes com os achados do estudo correlacional promovido.

O estudo das dissociações foi um princípio adotado pela "neuropsicologia cognitiva" para a investigação dos subcomponentes das funções cognitivas ${ }^{24}$. Na dissociação, constata-se uma diferença no desempenho em duas tarefas pelo mesmo indivíduo. A presença de dissociações entre as habilidades envolvidas nos testes de $\mathrm{PA}(\mathrm{C})$ e as funções neuropsicológicas investigadas confirmam e reforçam a hipótese da relação não causal existente entre déficits em funções neuropsicológicas e déficits perceptuais auditivos. Confirma, ainda, que possa haver diferença no desempenho de um paciente nos dois tipos de tarefas avaliadas e que pode haver prejuízos de desempenho em algumas tarefas em contraposição a um desempenho preservado em outras tarefas, o que confirma a evidência da existência de diferentes sistemas neurais subjacentes, isto é, de um processamento relativamente independente ${ }^{15}$. Este achado está de acordo com a definição de TPA(C) como sendo um déficit no processamento neural do estímulo acústico que não é resultado de uma disfunção em outras modalidades ${ }^{1}$. Apesar de neurônios da via auditiva no cérebro estabelecerem interconectividade com uma variedade de neurônios de outras áreas não auditivas, incluindo sistema límbico, giro do cíngulo, hipocampo e lobo frontal ${ }^{2}$, neurônios nas áreas auditivas podem não responder exclusivamente ao estímulo auditivo, mas também podem responder primariamente a ele ${ }^{5}$. No entanto, devese salientar que a ausência de uma relação causal não exclui a possibilidade de comorbidades entre déficits de processamento auditivo e prejuízos cognitivos específicos.

Alguns participantes não obtiveram desempenho abaixo do esperado para a idade em testes de $\mathrm{PA}(\mathrm{C})$ e apresentaram déficits em algumas funções neuropsicológicas e vice-versa. Na medida em que as funções com maior correlação com habilidades do $\mathrm{PA}(\mathrm{C})$ foram atenção, memória e linguagem, esperava-se que tais processos cognitivos apresentassem as dissociações menos frequentes. Tal hipótese foi confirmada, já que as funções neuropsicológicas como memória verbal episódica, fluência verbal e linguagem oral repetição foram as que menos se dissociaram com as tarefas de processamento auditivo. Isso pode sugerir que essas funções 
são as que mais compartilham mecanismos cognitivos subjacentes com as habilidades auditivas.

Por fim, quanto à terceira questão de pesquisa, "A análise intertestes do desempenho dos participantes na bateria selecionada para a avaliação do $\mathrm{PA}(\mathrm{C})$ quando comparada ao desempenho na avaliação neuropsicológica confirma os resultados encontrados para as questões anteriores?", a hipótese era de que esta análise qualitativa mais global confirmaria as relações encontradas nos achados em resposta às duas questões anteriores. Para o entendimento das dissociações verificadas, foi realizada uma análise inter e intratestes ${ }^{1} \mathrm{com}$ base nos resultados obtidos. Mediante tal interpretação, procurou-se, também, compreender o perfil geral de desempenho dos participantes. Essa análise é sugerida por alguns autores ${ }^{2,5,7,25-27}$ ao afirmarem que a interpretação dos testes deve ser ponto fundamental para compreender as dificuldades apresentadas pelo paciente em contrapartida à influência de fatores supramodais e a modalidade específica de processamento perceptual auditivo. A interpretação deve incluir a análise intrateste, que compara os padrões observados em determinado teste, fornecendo dados interpretativos, assim como a intertestes, caracterizada pela comparação entre os testes da bateria diagnóstica. Além disso, faz-se necessária a análise transdisciplinar, que consiste em comparar os resultados da avaliação de $\mathrm{PA}(\mathrm{C})$ e outros dados não audiológicos, como avaliação de linguagem e das funções cognitivas ${ }^{1}$.

Dos 12 participantes avaliados, quando submetidos a uma análise intra, intertestes e transdiciplinar. Quatro deles obtiveram resultados quantitativos que não são compatíveis com um déficit específico de $\mathrm{PA}(\mathrm{C})$, pois apresentaram falhas apenas nos testes PPST e SSI/MCI. Os resultados encontrados podem sugerir outro tipo de dificuldade, por exemplo, déficit na atenção, como sugerido por estes últimos autores. Eles afirmam que falhas apenas nestes tipos de testes não são consistentes para caracterizar um TPA $(C)$, podendo indicar que o baixo desempenho possa ocorrer por influência de fatores atencionais mais globais prejudicados 1,26-29.

Esta análise do perfil encontrado foi confrontada com o resultado obtido nas tarefas do Neupsilin. Assim, uma interpretação geral de desempenho em todo o instrumento foi também realizada, visando ao entendimento do perfil neuropsicológico do participante. Dois dos quatro adolescentes, cujos resultados na bateria de testes de $\mathrm{PA}(\mathrm{C})$ não foram compatíveis com uma disfunção auditiva central, apresentaram alguns déficits em subtestes do Neupslin que sugerem dificuldades atencionais, confirmando a hipótese levantada na avaliação de $\mathrm{PA}(\mathrm{C})$. Apesar de áreas auditivas do cérebro demonstrarem diferenças de funcionamento neuroanatômico em indivíduos portadores de transtorno de déficit de atenção/hiperatividade (TDAH), é possível distinguir a sintomatologia entre eles ${ }^{27,28}$. Embora as dificuldades de atenção frequentemente caracterizem o TPA(C) e o TDAH (particularmente os tipos combinado e o predominantemente desatento), há distinções a serem observadas na natureza da desatenção nos dois distúrbios ${ }^{3}$. Os déficits de atenção no TDAH tipicamente são pervasivos e supramodais e têm impacto em mais de uma modalidade sensorial ${ }^{28}$. Em oposição, indivíduos com TPA $(C)$ apresentam dificuldades de atenção que podem estar restritas à modalidade auditiva ${ }^{3}$. A desvantagem da orelha esquerda competitiva (EC) em testes dicóticos comumente percebida em indivíduos com TPA $(C)$, assim como um prejuízo nas condições de escuta contralateral ou ipsilateral como uma função do tronco encefálico, vão contra uma dificuldade de atenção pervasiva e contribuem para distinguir TPA(C) de TDA/H ${ }^{27,28}$. Esse dado reforça a importância da avaliação do $\mathrm{PA}(\mathrm{C})$ para o diagnóstico diferencial do TDA/H e da análise criteriosa de desempenho nos testes que avaliam a função auditiva central. Além disso, ressalta-se a necessidade da análise e entendimento sobre quais os testes que de fato seriam mais resistentes a disfunções da atenção e quais os que seriam os mais suscetíveis a elas ${ }^{30}$. Em complementaridade, ainda é possível afirmar que esses dois participantes fecharam critérios, pela análise geral do Neupsilin, para déficit de atenção e não apresentaram o perfil de desempenho nos testes de $\mathrm{PA}(\mathrm{C})$, sugerido pelos autores, para o diagnóstico diferencial dos dois quadros.

Além destes dois participantes, cujos resultados foram acima interpretados, os resultados da análise dos testes de outros dois adolescentes não confirmaram a hipótese de déficit de atenção, tendo apresentado o mesmo perfil de desempenho na avaliação de PA(C) (falha no SSI e no PPST). Estes adolescentes não apresentaram déficits no Neupslin que pudessem sugerir a presença de uma disfunção neuropsicológica. Pode-se inferir, então, que as falhas nos testes de $\mathrm{PA}(\mathrm{C})$ podem ter ocorrido por fadiga, diminuição do nível de atenção no momento da realização da tarefa, sem que a presença de um déficit de atenção propriamente dito estivesse presente.

Outros dois participantes, que completam o total de seis cujos resultados se mostraram particularmente interessantes para a análise qualitativa global em pauta, apresentaram resultados compatíveis com TPA $(C)$ na análise inter e intratestes, como falhas no SSW e SSI associadas ${ }^{1,27}$. Ambos relataram dificuldades em manter a atenção, mas em grau 
leve, nunca tendo procurado investigar tal queixa. $\mathrm{Na}$ análise do desempenho nos subtestes do Neupsilin, esses dois participantes não apresentaram déficits que confirmassem um quadro sugestivo de dificuldade de atenção e de dificuldades em habilidades neuropsicológicas de modo geral, mas foi confirmado o déficit perceptual auditivo através da análise do desempenho geral nos testes de PA(C). Esse achado corrobora a existência de uma relação de interdependência entre os processamentos cognitivos em geral e o processamento auditivo (central) ${ }^{2}$. Remete, também, ao entendimento sobre as queixas de desatenção, que por vezes podem levar a diagnósticos falso-positivo. No caso desses dois participantes, a hipótese de desatenção não foi confirmada pela avaliação neuropsicológica, mas compreendida pela presença de um déficit perceptual auditivo. $O$ fato dos dois testes que indicaram a presença de déficit em PA(C), SSW e SSI associados, apresentarem grande demanda da habilidade de figura-fundo auditivo pode justificar a queixa de desatenção desses dois participantes. Isso fortalece a importância do diagnóstico diferencial, pois nesse caso a desatenção está ligada ao comprometimento das funções auditivas centrais e não pela presença de um TDA/H.

A partir de uma análise do desempenho geral nos testes de $\mathrm{PA}(\mathrm{C})$ pode-se observar ainda que, os participantes que não fecharam critérios para comprovar um déficit perceptual auditivo, mas que apresentaram falhas combinadas nos testes - PPS e SSI/ MCl, apresentaram falhas também nos subtestes do Neupsilin cujas tarefas exigem a participação do executivo central (memória de trabalho, funções executivas). Esse fato chama a atenção e pode demonstrar que a participação do executivo central é relevante para tarefas de $\mathrm{PA}(\mathrm{C})$ envolvidas nos testes mencionados e está de acordo com a literatura que aponta a sobreposição de áreas cerebrais na realização de tarefas auditivas e cognitivas em geral ${ }^{5}$. Portanto, alguns cuidados ao avaliar pacientes com suspeita ou com a comprovação de um TDA/H devem ser tomados. Neste contexto, uma visão mais ampla, sob a perspectiva da neurociência, permite a verificação de outras dificuldades além das de $\mathrm{PA}(\mathrm{C})$ presentes no mesmo indivíduo e evidencia a necessidade da aplicação de testes adicionais em outras modalidades e para outras funções cognitivas para elucidar o diagnóstico ${ }^{30}$, mais especificamente testes auditivos e não auditivos ${ }^{10}$.

Ressalta-se, ainda, a necessidade de se explorar de modo mais profundo e detalhado o quão sensíveis são os testes PPST e SSI para dificuldades de atenção e o quão especifico é o teste SSW para determinar a presença de um TPA(C). Sugere-se isso porque na amostra do presente estudo os únicos participantes que falharam nesse teste, que foi determinante para sugerir a presença de déficits perceptuais auditivos, não apresentaram déficits nas habilidades do Neupsilin que sugerissem a presença de dificuldades neuropsicológicas.

Embora os achados preliminares desta investigação tenham sido elucidativos, mostram-se, ainda incipientes e restritos. É importante considerar que, apesar de rico em detalhes, foi utilizado um instrumento breve de avaliação neuropsicológica (Neupsilin) e, portanto, outros estudos devem ser promovidos com baterias completas. Pesquisas futuras devem abranger o exame da maior quantidade possível de componentes atencionais, mnemônicos e executivos, funções que apresentaram maior associação com as habilidades de $P A(C)$. Uma bateria breve oferece um panorama geral da cognição dos examinandos, mas não aprofunda a investigação de todos os componentes cognitivos, tal como o Neupsilin que não contempla a avaliação de atenção dividida direta e de funções executivas de maneira mais completa. Estas habilidades parecem estar envolvidas nas tarefas que avaliam a função auditiva central e auxiliariam na resolução de algumas questões.

Sugere-se a continuidade do estudo com o aumento da amostra e a aplicação de testes mais específicos por função cognitiva. Com o aumento das pesquisas em busca de um melhor entendimento das relações entre $\mathrm{PA}(\mathrm{C})$ e habilidades cognitivas, as dissociações e comorbidades poderão ser pormenorizadamente caracterizadas. De um modo geral, quanto a implicações clínicas diagnósticas, os achados do presente estudo sugerem a ausência de uma relação de causa-e-efeito entre déficits neuropsicológicos de funções específicas e $\operatorname{TPA}(\mathrm{C})$, que precisam ser melhor investigadas com a aplicação de mais testes ${ }^{31} \mathrm{e}$ utilizando recursos estatísticos mais apropriados. Em contrapartida, indica a ocorrência de uma comorbidade de déficits, em alguns casos, sugerindo a existência de uma sobreposição de habilidades cognitivas subjacentes a ambos os processamentos.

Um TPA(C) real e verdadeiro, comprovado, é caracterizado por um déficit que ocorre primariamente quando o individuo se ocupa da informação auditiva e não quando uma informação similar é apresentada em outras modalidades sensoriais (por exemplo, visuais, táteis, olfatórias, etc.). Essa abordagem exige que comparações relevantes de tarefas similares sejam feitas em no mínimo duas modalidades sensoriais separadas. Modalidades auditivas e visuais são escolhas lógicas para a comparação, porque ambas são canais principais para a comunicação. Podem ser úteis em determinar se os déficits perceptuais observados são específicos 
de modalidade ou não. Já que o TPA $(C)$ refere-se a déficits em processos perceptuais específicos auditivos, fatores não perceptuais auditivos precisam ser descartados antes que o diagnóstico seja estabelecido ${ }^{2}$. Além de medidas comportamentais, instrumentos e métodos diagnósticos, uma abordagem multidisciplinar de investigação que inclua métodos de neuroimagem, por exemplo, seriam procedimentos úteis na diferenciação de TPA(C) de outros transtornos do desenvolvimento cognitivo ${ }^{32}$.

Considerando o que os autores já sinalizavam a respeito do entendimento dos TPA(C) desde 1995, pressupostos ainda reafirmados em 2005, mas com um olhar mais amplo nos diagnósticos, entende-se a importância da avaliação de $\mathrm{PA}(\mathrm{C})$ como parte da avaliação neuropsicológica. Portanto, ressaltase que ambas devem ocorrer com o objetivo de que diagnósticos mais precisos e propostas de intervenção mais eficazes sejam promovidos. Ressalta-se a importância de pesquisas longitudinais com rigor metodológico para estudar melhor estas questões ${ }^{33}$.

\section{CONCLUSÃO}

Os resultados desse estudo evidenciam a necessidade de se repensar o contexto da administração dos testes de $\mathrm{PA}(\mathrm{C})$. Em geral, há uma rotina de aplicação desses testes como parte integrante da bateria audiológica e nem sempre a análise transdisciplinar é realizada para que os clínicos e avaliadores obtenham o entendimento de cada caso apoiados nos princípios da neurociência. Acreditase que a avaliação do PA(C) deveria ser analisada sob o viés neuropsicológico, assim como uma bateria neuropsicológica envolvendo estímulos verbais não poderia ser interpretada sem o conhecimento do status das habilidades de processamento auditivo. Nesse sentido, a avaliação de processamento auditivo deve ser administrada e interpretada em um contexto mais amplo da cognição, com raciocínio clínico neuropsicológico no desempenho dos testes que avaliam a função auditiva central. A análise da história clínica, avaliações neuropsicológicas e PA(C) devem andar juntas num processo de complementaridade em busca de uma maior garantia da acurácia de diagnóstico e da decisão de condutas terapêuticas.

Espera-se que os resultados iniciais neste artigo abordados sejam um alerta a profissionais que atuam em avaliação e reabilitação da função auditiva central sobre o cuidado com os critérios diagnósticos utilizados.

\section{AGRADECIMENTOS}

Dedicamos um especial agradecimento a Fonoaudióloga Sabrina Scherer pela importante contribuição nos procedimentos de avaliação audiológica dos participantes desta pesquisa.

\begin{abstract}
Purpose: to check if there is a relation between the performance in auditory processing tests and in cognitive tasks. Methods: the sample was made up by 12 teenagers in typical development, all of them high-school students (third year) in private schools from Rio Grande do Sul, Brazil. They were evaluated through an auditory processing battery as well as with the Brazilian Brief Neuropsychological Assessment Battery NEUPSILIN. Data analyses included Person's correlation and qualitative interests interpretation towards a comparison of the performance in (C)AP's battery and in the neuropsychological assessment. Results: regarding (C)AP's battery, SSI - MCI - Synthetic Sentence Test with ipsilateral competitive message, SSW - Test of Alternated Disyllables and Digit Dicotics tests showed a significant strong positive correlation with NEUPSILIN's subtests that follows: attention - reverse counting, face perception, oral language - automated language and repetition, episodic verbal memory and working memory - auditory word span in sentences. Some (C)AP's tests presented a greater frequency of dissociation when compared to neuropsychological tasks. Correlation index suggest that (C)AP's tests and neuropsychological tasks seem to evaluate some common basic cognitive abilities. The observed dissociation suggest that auditory and neuropsychological processing are partially independent on each other. The interests analysis confirms these results. Conclusion: there is a need to rethink about the administration of (C)AP's tests, when it comes to the addition relation between neuropsychological assessment and auditory processing examination.
\end{abstract}

KEYWORDS: Auditory Perception; Neuropsychological Tests; Neuropsychology; Adolescent 


\section{REFERÊNCIAS}

1. American Speech-Language-Hearing Association - ASHA. (Central) Auditory processing disorders: technical report. 2005. [acesso em: agosto 2005]. Disponível em: URL: http: //www.asha.org/ members/deskref-journals/deskref/default.

2. Bellis TJ. Assessment and management of central auditory processing disorders in the educational setting: from science to practice. San Diego, USA: Singular Publishing Group; 2003.

3. Musiek FE, Chermak DD. Handbook of (central) auditory processing disorders: auditory neuroscience and clinical diagnosis. Vol. 1. San Diego, USA: Plural Publishing; 2007.

4. Moore DR. Auditory processing disorder (APD): definition, diagnosis, neural basis, and intervention. Audiol Med. 2006; 4(1):4-11. http://dx.doi. org/10.1080/16513860600568573

5. Musiek FE, Bellis TJ, Chermak GD. Nonmodularity of the central auditory nervous system: implications for (central) auditory processing disorder. Am $J$ Audiol. 2005; 14(2):128-38. http://dx.doi. org/10.1044/1059-0889(2005/014)

6. Cacace AT, McFarland DJ. Central auditory processing disorder in school-aged children: a critical review. J Speech Lang Hear Res. 1998; 41(2):355-73.

7. Cacace AT, McFarland DJ. The importance of modality specificity in diagnosing central auditory processing disorder. Am J Audiol. 2005; 14(2):112-23.

8. Rosen S. "A riddle wrapped in a mystery inside an enigma". Defining central auditory processing disorder. Am J Audiol. 2005; 14(2):139-42. http:// dx.doi.org/10.1044/1059-0889(2005/015)

9. Jerger J, Musiek F. Report of the consensus conference on the diagnosis of auditory processing disorders in school-age children. J Am Acad Audiol. 2000; 11(9):467-74.

10. Bellis TJ, Billiet C, Ross J. Hemispheric lateralization of bilaterally presented homologous visual and auditory stimuli in normal adults, normal children, and children with central auditory dysfunction. Brain Cognition. 2008; 66(3):280-9.

11. Lezak MD, Howieson DB, Loring DW. Neuropsychological assessment. 4. ed. New York, USA: Oxford; 2004. p. 375-413.

12. Willmes K. Methodological and statistical considerations in cognitive neurolinguistics. In: Stemmer B, Whitaker HA, organizadores. Handbook of neurolinguistics. San Diego, USA: Academic Press; 1998. p. 57-70.

13. Critério de Classificação Econômica Brasil CCEB. [homepage na internet] [acesso em maio
2007]. Disponível em: URL: http://www.abep.org/ codigosguias/ABEP_CCEB_2003.pdf.

14. Auditec. Inc. St. Louis: MO USA. Disponível em: URL: http://www.auditec.com

15. Pereira LD, Schochat E. Processamento auditivo central: manual de avaliação. São Paulo: Lovise; 1997.

16. Pawlowski J. Evidências de validade e fidedignidade do instrumento de avaliação neuropsicológica breve Neupsilin. [dissertação]. Porto Alegre (RS): Universidade Federal do Rio Grande do Sul; 2007.

17. Amaral RA, Malbergier A. Avaliação de instrumento de detecção de problemas relacionados ao uso do álcool (CAGE) entre trabalhadores da Prefeitura do Campus da Universidade de São Paulo (USP) - Campus Capital. Rev Bras Psiquiatr. 2004; 26(3):156-63.

18. Fonseca RP, Salles JF, Parente MAMP. Development and content validity of the Brazilian brief neuropsychological assessment battery Neupsilin. Psychol Neurosci. 2008; 1(1):55-62.

19. Fonseca RP, Salles JF, Parente MAMP. Instrumento de avaliação neuropsicológica breve: Neupsilin. São Paulo: Vetor; 2009.

20. Nijland L. Speech perception in children with speech output disorders. Clin Linguist Phonet. Mar 2009; 23(3):222-39.

21. Bamiou DE, Sisodiya S, Musiek FE, Luxon LM. The role of the interhemispheric pathway in hearing. Brain Res Rev. 2007; 56(1):170-82.

22. Baddeley A. Working memory and language: an overview. J Commun Disord. 2003; 36(3):189-208.

23. Strauss E, Sherman EMS, Spreen O. A compendium of neuropsychological tests. 3. ed. New York, USA: Oxford; 2006.

24. McCarthy RA, Warrington EK. Cognitive neuropsychology: a clinical introduction. London, UK: Academic Press Ltd; 1990.

25. Shinn JB, Baran JA, Moncrieff DW, Musiek FE. Differential attention effects on dichotic listening. $J$ Am Acad Audiol. 2005; 16(4):205-18.

26. Katz J, Tillery KL. Can central auditory processing tests resist supramodal influences? Am J Audiol. 2005; 14(2):124-7.

27. Riccio CA, Cohen MJ, Garrison T, Smith B. Auditory processing measures: correlation with neuropsychological measures of attention, memory and behavior. Child Neuropsychol. 2005; 11(4):363-72.

28. Chermak GD, Hall JW III, Musiek FE. Differential diagnosis and management of central auditory processing disorder and attention deficit hyperactivity disorder. J Am Acad Audiol. 1999; 10(6):289-303. 
29. Chermak GD. It takes a team to differentially diagnose APD. Hear J. 2003; 56(4):71. http://dx.doi. org/10.1097/01.HJ.0000293914.81047.29

30. Dawes P, Bishop D. Auditory processing disorder in relation to developmental disorders of language, communication and attention: a review and critique. Int J Lang Commun Disord. jul-aug 2009; 44(4):44065. http://dx.doi.org/10.1080/13682820902929073 31. Bamiou DE, Campbell N, Sirimanna T. Management of auditory processing disorders. Audioll Med. 2006; 4(1):46-56.
32. Kim MJ, Jeon HA, Lee KM, Son YD, Kim YB, Cho $\mathrm{ZH}$. Neuroimaging features in a case of developmental central auditory processing disorder. J Neurol Sci. 2009; 277(1-2):176-80.

33. White RF, Campbell R, Echeverria D, Knox SS, Janulewicz P. Assessment of neuropsychological trajectories in longitudinal population-based studies of children. J Epidemiol Commun Health. 2009; 63(Suppl1):i15-26.

DOI: 10.1590/S1516-18462010005000027

RECEBIDO EM: 29/05/2009

ACEITO EM: 06/12/2009

Endereço para correspondência:

Mirella Liberatore Prando

Rua Quintino Bocaiúva, 1410

Porto Alegre - RS

CEP: 90440-050

E-mail: mirellalprando@gmail.com 